\title{
Research on Course Group Construction of Information Management and Information System Specialty in Big Data Era
}

\author{
Xiqin $\mathrm{Ao}^{1, \mathrm{a},{ }^{*}}$, Weiwei $\mathrm{Wu}^{1, \mathrm{~b}}$ and Lina Tao ${ }^{1, \mathrm{c}}$ \\ ${ }^{1}$ Institute of information engineering, Anhui Xinhua University, Anhui, 230088, China \\ aaoxiqin@axhu.edu.cn, bwuweiwei@axhu.edu.cn, ctaolina@axhu.edu.cn \\ ${ }^{*}$ Corresponding author
} Keywords: Big Data, Information Management and Information System, Relevance, Course
Group.

\begin{abstract}
In order to meet the needs of information management and information professionals in the era of big data, the correlation analysis and relevance mining were carried out to investigate the correlation and relevance between courses. On this basis, combining ability training with course teaching, and combining with the requirement of information management and information system students'ability in the era of big data, a corresponding professional curriculum group was formed. The research results can provide a basis for the formulation of talent training program and professional reform of information management and information system specialty.
\end{abstract}

\section{大数据时代信息管理与信息系统专业课程群建设研究 \\ 敖希琴 $1, a,{ }^{*}$ ，吴炜炜 $1, \mathrm{~b},{ }^{*}$ ，陶丽娜 $1, \mathrm{c}$ \\ 1安徽新华学院信息工程学院, 合肥, 安徽, 中国 \\ aaoxiqin@axhu.edu.cn, bwuweiwei@axhu.edu.cn, ctaolina@axhu.edu.cnl \\ ”通讯作者}

关键词:大数据; 信息管理与信息系统; 关联性; 课程群

摘要. 为适应大数据时代对信息管理与信息专业的人才的需求, 通过对信息管理与信息系统专 业学生成绩进行相关性分析和关联性挖掘, 以考察课程间相关性和关联性。在此基础上, 将 能力培养和课程教学结合一起, 并结合大数据时代对信息管理与信息系统专业学生能力的要 求, 形成相应的专业课程群。研究结果可为信息管理与信息系统专业人才培养方案制定和专 业改革提供依据。

\section{1. 引言}

随着计算机和信息通信技术的发展, 大数据的应用日益渗透到社会的各个方面 ${ }^{[1-2]}$ 。大数 据正在激活人们从数据中挖掘价值, 引发人们从数据中 “钻取石油、开采黄金”。市场竞争 日渐复杂, 对未来发展趋势的判断和预测能力成为企业的核心竞争力所在, 然而现有的数据 分析方法已经不能完全满足大数据时代企业的需求 ${ }^{[3-4]}$ 。因此, 国内外相关高等院校和高新技 术企业为应对大数据的发展和挑战, 纷纷提出了大数据高端人才的培养措施和方案 ${ }^{[5-6]}$ 。作为 与大数据应用最为相关的专业之一，信息管理与信息系统专业 (以下简称 “信管” 专业) 应 该以大数据发展为契机, 改革专业人才培养方案, 以培养更多符合社会需求的复合型人才。 


\section{2. 大数据时代信管专业能力培养目标}

国家教育部发布的《普通高等院校本科专业目录和专业简介（2012年）》中关于信管专 业的培养目标描述为：具备良好的数理基础、管理学和经济学理论知识、信息技术知识及应 用能力，掌握信息系统的规划、分析、设计、实施和管理等方面的方法与技术，具有一定的 信息系统和信息资源开发利用实践和研究能力的复合型高级专门人才 ${ }^{[7]}$ 。

学生应具备的能力: (1)掌握信息管理和信息系统的基本理论、基本知识; (2)掌握管理信 息系统的分析方法、设计方法和实现技术; (3)具有信息组织、分析研究、传播与开发利用的 基本能力; (4)具有综合运用所学知识分析和解决问题的基本能力; (5)掌握文献检索、资料查 询、收集的基本方法，具有一定的科研和实际上作能力。

\section{3. 课程关联性研究}

\section{1 数据来源与预处理}

为保证研究的科学性和严谨性, 本课题的实验数据来源于学校教务系统数据库, 为我校 信管专业11 14届学生大学期间的所有课程的真实成绩, 原始数据共227条记录。由于2位学生 成绩由于缺考, 成绩存在缺失值, 对这两位学生的成绩进行了删除处理, 有效数据225条。

由于部分学生有挂科情况, 因此出现一门课程有两个成绩：正考成绩和补考成绩，为保 证研究的准确性, 均选用了正考成绩作为分析对象。所有的课程被分为两大类: 必修课和选 修课，每个学生的选修课程不同，评分标准不同，因此仅将必修课程作为分析对象。

\section{2 课程相关性分析}

相关分析，是通过定量的指标，来描述事物之间联系的强弱程度。本课题中课程成绩有 两种计分方式: 百分制和等级制。百分制成绩属于连续性变量，因此这类课程选用了Pearson 相关系数来表示课程间的相关性大小; 等级制成绩属于有序变量, 因此这类课程选用了 Kendall's Tau-c相关系数来表示课程间相关性的大小。

对所有课程进行了两两相关性分析，相关性分析结果显示同类课程的成绩关联性最强， 例如大学英语类课程、数学类课程和部分计算机类课程相关系数较大。此结果与实际情况及 认知也是完全相符的。由于英语类、数学类课程属于通识类课程, 该类课程由学校统一规划 和安排, 跟专业教学目标关联性不大, 因此此类课程不作为重点研究分析对象。本课题的主 要研究对象为信管专业的专业课程, 综合考虑, 从所有课程中筛选出了 $27 门$ 门课程作为研究分 析对象。

对专业课中所有计算机类课程进行相关性分析，结果如表1所示，大部分课程之间都有较 强的相关性，但也有一些课程与其他课程间的关联性较弱，例如动态网站设计与架构这门课 程与其他课程间的相关性较小。

表1 计算机类课程相关性

\begin{tabular}{|c|c|c|c|c|c|c|c|c|c|c|}
\hline & $\begin{array}{c}\text { 计算机应用 } \\
\text { 基础与程序 } \\
\text { 设计 }\end{array}$ & 数据结构 & $\begin{array}{l}\text { 面向对象设 } \\
\text { 计方法C++ }\end{array}$ & $\begin{array}{c}\text { 数据库原理 } \\
\text { 与应用 }\end{array}$ & $\begin{array}{c}\text { 管理信息系 } \\
\text { 统 }\end{array}$ & $\begin{array}{c}\text { MATLAB程序 } \\
\text { 设计 }\end{array}$ & 计算机网络 & 软件工程 & $\begin{array}{c}\text { JAVA程序设 } \\
\text { 计 } \mathrm{B}\end{array}$ & $\begin{array}{l}\text { 动态网 } \\
\text { 站设计 } \\
\text { 与架构 }\end{array}$ \\
\hline $\begin{array}{c}\text { 计算机应用 } \\
\text { 基础与程序 } \\
\text { 设计 } \\
\end{array}$ & 1 & -0.022 & 0.091 & 0.073 & 0.064 & $.253^{* *}$ & -0.036 & -0.092 & $.232 * *$ & 0.060 \\
\hline 数据结构 & -0.022 & 1 & $.539 * *$ & $.496^{* *}$ & $.376^{* *}$ & $.175^{* *}$ & $.303 * *$ & $.200^{* *}$ & 0.044 & 0.055 \\
\hline $\begin{array}{c}\begin{array}{c}\text { 面向对象设 } \\
\text { 计方法 } \mathrm{C}++\end{array} \\
\end{array}$ & 0.091 & $.539 * *$ & 1 & $.528 * *$ & $.416^{* *}$ & $.188^{* *}$ & $.313 * *$ & 0.092 & $.195^{* *}$ & 0.054 \\
\hline $\begin{array}{c}\text { 数据库原理 } \\
\text { 与应用 }\end{array}$ & 0.073 & $.496^{* *}$ & $.528 * *$ & 1 & $.584 * *$ & $.262 * *$ & $.277^{* *}$ & 0.016 & 0.102 & -0.006 \\
\hline $\begin{array}{c}\text { 管理信息系 } \\
\text { 统A }\end{array}$ & 0.064 & $.376^{* *}$ & $.416^{* *}$ & $.584^{* *}$ & 1 & $.266^{* *}$ & $.255^{* *}$ & 0.056 & 0.123 & 0.034 \\
\hline $\begin{array}{l}\text { MATLAB程序 } \\
\text { 设计 }\end{array}$ & $.253 * *$ & $.175^{* *}$ & $.188 * *$ & $.262 * *$ & $.266^{* *}$ & 1 & $.200^{* *}$ & $.163^{*}$ & $.227 * *$ & 0.105 \\
\hline
\end{tabular}




\begin{tabular}{|c|c|c|c|c|c|c|c|c|c|c|}
\hline 计算机网络 & -0.036 & $.303^{* *}$ & $.313^{* *}$ & $.277^{* *}$ & $.255^{* *}$ & $.200^{* *}$ & 1 & $.149^{*}$ & $.175^{* *}$ & 0.102 \\
\hline 软件工程 & -0.092 & $.200^{* *}$ & 0.092 & 0.016 & 0.056 & $.163^{*}$ & $.149^{*}$ & 1 & 0.040 & $.181^{* *}$ \\
\hline $\begin{array}{c}\text { JAVA程序设 } \\
\text { 计 }\end{array}$ & $.232^{* *}$ & 0.044 & $.195^{* *}$ & 0.102 & 0.123 & $.227^{* *}$ & $.175^{* *}$ & 0.040 & 1 & $.340^{* *}$ \\
\hline $\begin{array}{c}\text { 动态网站设 } \\
\text { 计与架构 }\end{array}$ & 0.060 & 0.055 & 0.054 & -0.006 & 0.034 & 0.105 & 0.102 & $.181^{* *}$ & $.340^{* *}$ & 1 \\
\hline
\end{tabular}

对专业课中所有管理类课程进行相关性分析，结果如表2所示，只有《生产与运作管理与 信息管理概论》两门课程间无显著关联性, 其他所有课程都显著相关, 其中《管理学原理》 和其他课程间的关联性最强。

表2 管理类相关性

\begin{tabular}{|c|c|c|c|c|c|c|c|c|}
\hline & $\begin{array}{c}\text { 信息管理概 } \\
\text { 论 }\end{array}$ & $\begin{array}{c}\text { 管理学原 } \\
\text { 理A }\end{array}$ & $\begin{array}{c}\text { 生产与运作管 } \\
\text { 理 }\end{array}$ & $\begin{array}{l}\text { 物流与供应链 } \\
\text { 管理 }\end{array}$ & $\begin{array}{c}\text { 人力资源管理 } \\
\mathrm{A}\end{array}$ & $\begin{array}{c}\text { 企业资源计划 } \\
\text {-ERP }\end{array}$ & 决策支持系统 & $\begin{array}{c}\text { 管理信息系统 } \\
\text { A }\end{array}$ \\
\hline 信息管理概论 & 1 & $.562^{* *}$ & 0.122 & $.206^{* *}$ & $.442^{* *}$ & $.391^{* *}$ & $.219^{* *}$ & $.339^{* *}$ \\
\hline 管理学原理A & $.562^{* *}$ & 1 & $.192^{* *}$ & $.191^{* *}$ & $.493^{* *}$ & $.535^{* *}$ & $.201^{* *}$ & $.410^{* *}$ \\
\hline 生产与运作管理 & 0.122 & $.192^{* *}$ & 1 & $.271^{* *}$ & $.295^{* *}$ & $.285^{* *}$ & $.172^{* *}$ & $.291^{* *}$ \\
\hline $\begin{array}{c}\text { 物流与供应链管 } \\
\text { 理 }\end{array}$ & $.206^{* *}$ & $.191^{* *}$ & $.271^{* *}$ & 1 & $.446^{* *}$ & $.283^{* *}$ & $.265^{* *}$ & $.542^{* *}$ \\
\hline 人力资源管理A & $.442^{* *}$ & $.493^{* *}$ & $.295^{* *}$ & $.446^{* *}$ & 1 & $.410^{* *}$ & $.286^{* *}$ & $.545^{* *}$ \\
\hline $\begin{array}{c}\text { 企业资源计划 } \\
\text {-ERP }\end{array}$ & $.391^{* *}$ & $.535^{* *}$ & $.285^{* *}$ & $.283^{* *}$ & $.410^{* *}$ & 1 & $.274^{* *}$ & $.332^{* *}$ \\
\hline 决策支持系统 & $.219^{* *}$ & $.201^{* *}$ & $.172^{* *}$ & $.265^{* *}$ & $.286^{* *}$ & $.274^{* * *}$ & 1 & $.246^{* *}$ \\
\hline 管理信息系统A & $.339^{* *}$ & $.410^{* *}$ & $.291^{* *}$ & $.542^{* *}$ & $.545^{* *}$ & $.332^{* *}$ & $.246^{* *}$ & 1 \\
\hline
\end{tabular}

\section{3 基于Apriori算法的课程关联性分析}

关联分析的目的是要寻找事物之间的联系和规律，发现它们之间的关联关系 ${ }^{[8]}$ 。相关性 分析结果表示很多课程之间有较强的相关性, 为研究课程在开设时间上是否有一定的规律性, 因此选用了Apriori算法对课程的关联性进行分析。具有较高支持度和置信度的课程关联性分 析结果如表3所示:

表3 课程关联性分析结果

\begin{tabular}{|c|c|c|c|c|}
\hline 规则 & 前项 & 后项 & 支持度 \% & 置信度 \% \\
\hline 1 & 概率论与数理统计\&线性代数 & 离散数学 & 11.11 & 92.0 \\
\hline 2 & 数据结构 & 数据结构课程设计 & 30.22 & 83.82 \\
\hline 3 & $\begin{array}{c}\text { 数据结构 \&操作系统原理\& JAVA 程序 } \\
\text { 设计 }\end{array}$ & 动态网站设计与架构 B & 12.89 & 93.10 \\
\hline 4 & JAVA 程序设计 B & 动态网站设计与架构 B & 61.33 & 74.637 \\
\hline 5 & 数据结构 & 动态网站设计与架构 B & 30.22 & 73.53 \\
\hline 6 & 操作系统原理 & 动态网站设计与架构 B & 38.22 & 73.26 \\
\hline 7 & 操作系统原理 & JAVA 程序设计 B & 38.22 & 72.09 \\
\hline 8 & 面向对象设计方法 C++ & JAVA 程序设计 B & 33.33 & 70.67 \\
\hline 9 & 管理学原理 A\& & 生产与运作管理 & 14.67 & 93.94 \\
\hline 10 & 人资源管理 A & 物流与供应链管理 & 11.11 & 88 \\
\hline 11 & 人力资源管理 A & 运管学 A\& & 8.44 & 78.95 \\
\hline 12 & 企业资源计划-ERP & 年持系统 & 8.44 & 78.95 \\
\hline 13 & 运筹学 A\&资源计划-ERP & 8.44 & 73.68 \\
\hline
\end{tabular}


由表3关联规则挖掘结果可知: (1)同种类别的课程关联性较大: 计算机类课程、数学类课 程、管理类课程间都有较强的关联性。这与 3.2 中相关性分析结合完全一致, 在此就不一一赘 述。(2)先修课程的成绩对后续课程的成绩影响较大, 例如, 如果《数据结构》、《操作系统 原理》、 《JAVA程序设计》三门课程成绩都为优秀, 那么 《动态网站设计与架构B》成绩也

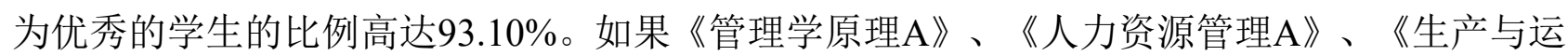
作管理》三门课程成绩都为优秀, 《生产与运作管理》也为优秀的学生比例高达 $93.94 \%$ 。这 都说明先修课程是基础, 基础掌握是否牢固直接影响后续课程的学习效果。因此, 在课程设 置时，应考虑该课程相关的先修课程是否已经完成。

\section{4. 课程群集成}

由上述关联分析结果可知，同类课程间具有较强的关联性，且课程设置的先后顺序很重 要。基于此, 在主张 “知识+实践=能力” 的培养理念提前下, 结合课程与课程的之间的关联 性, 将课程划分为基础能力、综合能力、核心能力和创新能力四大类, 并对每个大类的能力 进行细分, 将能力培养和课程教学结合一起, 形成相应的能力培养课程群。在此基础上, 对 大数据时代的信息收集、存储、分析、评价的方法和所使用的工具进行调研, 理清新时代信 管人才所应掌握技术和方法, 将这些技术和方法所对应的前沿课程融入到课程群中, 替换掉 同种类型较为老旧的课程，形成最终的课程群，如图1所示。

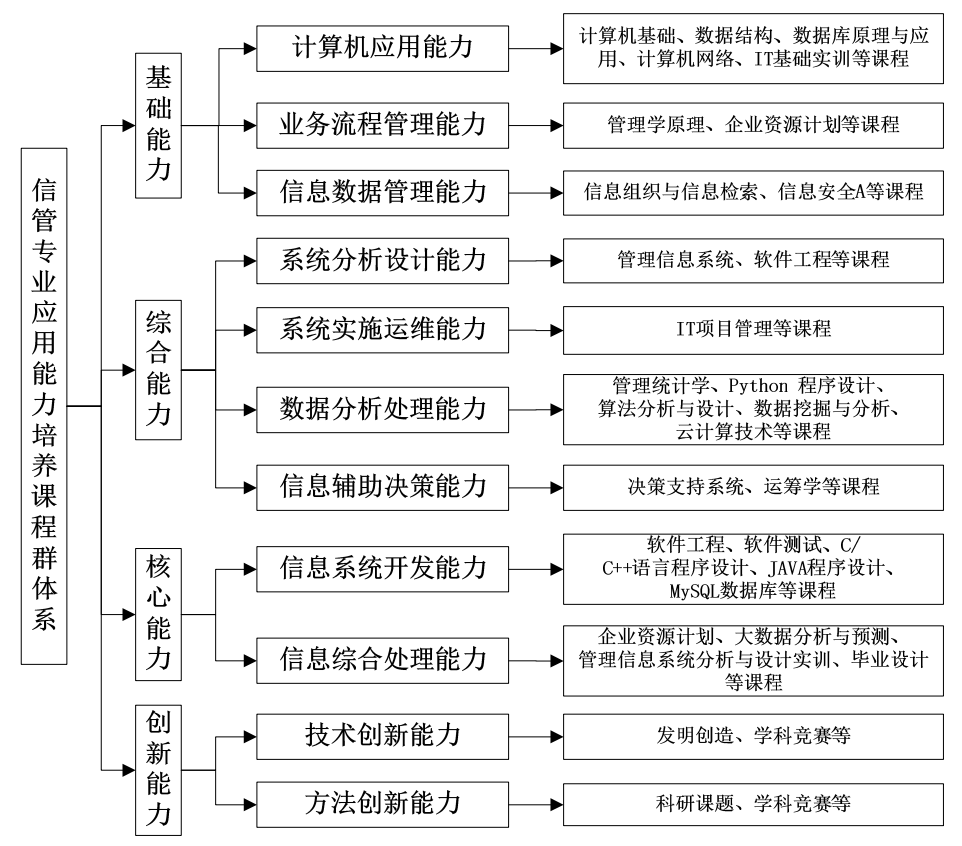

图 1 信管专业应用能力培养课程群体系

\section{5. 结束语}

本文通过学校教务系统收集了信管专业 $11^{\sim} 14$ 届学生大学期间所有课程的真实成绩, 并完 成了对成绩的相关性分析、关联性挖掘, 得到结论: 同种类别的课程成绩相关性较大; 先修 课程的成绩对后续课程的成绩影响较大。在此基础上, 将能力培养和课程教学结合, 并融合 大数据时代对信管专业学生能力的要求, 形成基础能力、综合能力、核心能力和创新能力, 四大能力培养的课程群。 


\section{致谢}

本文为2017年安徽省省级教学研究项目 《大数据时代的信息管理与信息系统专业课程群 建设研究》(No.2017jyxm0525)和2017年信管专业校级教学团队项目（No.2017jxtdx07）成果 之一。

\section{References}

[1] Lu Chenggong and Wei Yashuang. Research on cultivation mode of big data analysis talents in information management and information system, Computer Knowledge and Technology, vol.14, pp. 123-125+161, 2018.

[2] Ye Fulan. Research on the training model of applied talents of information management and information system specialty based on competence standard --- take Fuzhou institute of foreign languages and trade as an example, China Management Informatization, vol.20, pp. 239-241, 2017.

[3] Fang Jian. Research on computer information processing technology in the age of big data, Computer Products and Circulation, vol.06, pp.142, 2019.

[4] Zeng Weijia and Qin Fang. Research on the reform of applied course in information management and information system under big data, China Computer \& Communication, vol.02, pp.231-232, 2017.

[5] Cheng Yuepeng, Fan Guangyu and Li Shuo. Exploration and practice of information management major talent cultivation mode based on CDIO, Education Modernization, vol.06, pp.26-29+35, 2019.

[6] Yi Yanhong, Zhang Jing and Zhang Cong. Reform of big data direction course in information management and information systems for applied undergraduates, Research on Library Science, vol.07, pp. 15-20, 2019.

[7] Zhang Ning and Yuan Qinjian. Course construction and reform of information management and information system for data science, Journal of Modern Information, vol.37, pp.106-110+160, 2017.

[8] Ao Xiqin, Fei Jiulong and Chen Jiali. Research on college Students'Achievement analysis based on association rules, Education Modernization, vol.04, pp.240-244, 2017. 